\title{
Drug-drug-gene interactions and adverse drug reactions
}

\author{
Mustafa Adnan Malki ${ }^{1} \cdot$ Ewan Robert Pearson ${ }^{1}$
}

Received: 5 November 2018 / Revised: 11 November 2019 / Accepted: 17 November 2019 / Published online: 3 December 2019

(c) The Author(s) 2019. This article is published with open access

\begin{abstract}
The economic and health burden caused by adverse drug reactions has increased dramatically in the last few years. This is likely to be mediated by increasing polypharmacy, which increases the likelihood for drug-drug interactions. Tools utilized by healthcare practitioners to flag potential adverse drug reactions secondary to drug-drug interactions ignore individual genetic variation, which has the potential to markedly alter the severity of these interactions. To date there have been limited published studies on impact of genetic variation on drug-drug interactions. In this review, we establish a detailed classification for pharmacokinetic drug-drug-gene interactions, and give examples from the literature that support this approach. The increasing availability of real-world drug outcome data linked to genetic bioresources is likely to enable the discovery of previously unrecognized, clinically important drug-drug-gene interactions.
\end{abstract}

\section{Introduction}

It was previously and alarmingly reported that adverse drug reactions (ADRs) represent the fourth leading cause of death in the USA [1]. A recent review (2015) showed that $3.6 \%$ of patients were admitted to hospitals in Europe due to ADRs and $10 \%$ of patients developed side effects during their inpatient stay [2]. The latest report issued by MiDatabank in cooperation with the Medicines and Healthcare Products Regulatory Agency, shows an increasing trend in the number of reported ADRs in the period between 2011 and 2016 across the UK [3]. It has also been estimated that ADRs alone cost the NHS $£ 770 \mathrm{M}$ annually [4]. Nonsteroidal antiinflammatory drugs, diuretics, anticoagulants, and antiplatelets have been recognized to be the major culprits, with prescribing errors being major contributors to medication-related adverse events [5]. The chance of these errors increases when patients undergo multiple treatments; a situation that is highly prevalent in elderly patients [6]. There are a number of factors that influence the occurrence of ADRs secondary to

Supplementary information The online version of this article (https:// doi.org/10.1038/s41397-019-0122-0) contains supplementary material, which is available to authorized users.

Ewan Robert Pearson

e.z.pearson@dundee.ac.uk

1 Population Health \& Genomics, School of Medicine, University of Dundee, Dundee, UK drug-drug interactions, such as age, renal function, and other comorbidities. In addition, genetic variation is likely to play a crucial role in the development of ADRs. For example, when only considering genetic polymorphisms in three drug metabolizing enzymes (cytochrome P450 2C9 (CYP2C9), CYP2C19, and CYP2D6), $15 \%$ of the ADRs were due to drug-gene interactions, and $19 \%$ were due to drug-drug-gene interactions [7]. Incorporation of these gene variants increased the number of predicted clinically critical drug interactions by $\sim 51 \%$ [7]. Given the large number of genes involved in drug metabolism and transport, we cannot underestimate the importance of genetic variation in contributing to potential for clinically critical ADRs.

Following the recent advances in pharmacogenomics, the traditional view of drug-drug interactions needs to be modified to include genetic variation. To date the literature on drug-drug-gene interactions (DDGIs) is limited, with only one previous review evaluating the impact of CYP2C9, C19, and 2D6 variants [8]. In this review, we attempt to provide an in-depth framework for the classification of pharmacokinetic DDGIs caused by different mechanisms, and their potential impact to increase clinically critical drug interactions in the context of the polypharmacy seen in modern medicine today.

\section{Drug-drug-gene interactions}

DDGIs can be divided into three main categories: inhibitory interactions, induction interactions, and phenoconversion 
interactions. Inhibitory and induction interactions can be defined as any interactions that affect the victim drug's pharmacokinetics (PK) to increase or reduce concentrations of the drug, respectively. Induction or inhibition can occur either with the administration of a perpetrator drug that alters the victim drug metabolism or transport, or with the presence of loss- or gain-of-function (LOF or GOF) genetic variants that alter function of enzymes that alter metabolism or transport of the victim drug, or the combination of both. A DDGI can be thought of as a double hit-whereby the genetic variant and the perpetrator drug combine to act on transporter or metabolism pathways to greatly alter drug concentrations. It is also possible to see phenoconversionwhere the interacting drug effect and the genotype have opposing effects, resulting in a temporary phenotype shift e.g. neutralizing/reversing the effect of a GOF genotype when an inhibitory drug is prescribed. In this review we describe, with examples, different cases of interactions under each of the above three categories, focusing initially on metabolizing enzymes, before considering drug transporters.

\section{Drug-drug-metabolizing enzyme gene interactions (DDMEGIs)}

\section{Inhibitory interactions}

Inhibitory effects of drugs and genotype can alter substrate metabolism by both drug and genotype impacting on the same metabolizing enzyme, or on two distinct routes of metabolism.

In general, poor metabolizers are expected to experience the highest substrate drug plasma concentration, compared with other genotypes, when co-treated with inhibitors. For example, co-administration of simvastatin (a CYP2C9 inhibitor) with warfarin (CYP2C9 substrate) has been shown to reduce warfarin dosage requirements in CYP2C $9 * 3$ carriers with a greater percentage as compared with noncarriers (29\% vs 5\% respectively) [9]. A similar conclusion has been reported with celecoxib (Supplementary Table 1) [10]. The inhibitory effect of drug and genotype is not always additive-genetically poor metabolizers may have only limited further enzyme inhibition by administration of an inhibitory drug. For instance, a statistically significant elevation in rabeprazole (a CYP2C19 substrate) plasma levels was observed in both normal metabolizers and heterozygous genotype carriers after treatment with fluvoxamine (a CYP2C19 inhibitor) while no additional clinically significant elevation was detected with poor metabolizers who have already experienced the highest rabeprazole plasma levels [11]. A similar scenario is seen with other examples (Supplementary Table 1) [12-15].
Where a drug is metabolized by two or more CYP enzymes, then inhibition of one of these enzymes alone (by drug or genotype) may have minimal effect, due to redundancy of the pathways. However, if a genotype and interacting drug affect these different routes of metabolism, then the interaction may be very large. For example, it has been observed that for voriconazole (a CYP2C19 and CYP3A4 substrate) bioavailability is increased markedly ( 5.6-fold) in patients who have reduced CYP2C19 activity and are administered with atazanavir or ritonavir (potent CYP3A4 inhibitors) [16]. A similar scenario can be noted with other examples (Supplementary Table 1) [17-19].

Prodrugs, on the other hand, require the function of certain CYPs to be therapeutically active, and in these cases the effect is the opposite to that described above. Clopidogrel, for example, is activated by CYP1A2, CYP2B6, CYP3A4, CYP2C9, and CYP2C19 [20]. Carriers of LOF variants in one or more of these genes and co-administered with their inhibitors are at increased risk for treatment resistance. For instance, carriers of CYP2C19*2 and/or *3 alleles who are treated with clopidogrel and proton pump inhibitors (CYP2C19 inhibitors) were observed to be more likely to have reduced clopidogrel efficacy; the addition of a third risk factor (e.g., calcium channel blockers (CYP3A4 inhibitors)) was also correlated with a greater reduction in efficacy of clopidogrel [21, 22].

Figure 1 shows the predicted changes of plasma levels of active drugs and active metabolites of prodrugs with and without the presence of inhibitors and/or LOF variants.

\section{Induction interactions}

Increased metabolism of active drugs by an enzyme inducer or GOF variant will result in reduced efficacy of the victim drug. For example, when voriconazole (a CYP2C19 substrate) is co-prescribed with carbamazepine (CYP2C19 inducer) the voriconazole dose is usually increased to overcome this increased metabolism. In a case report, therapeutic concentrations of voriconazole were not achieved, as the patient carried two GOF CYP2C19 *17 variants [23].

The opposite effect is seen with prodrugs. Increased metabolism by an enzyme inducing drug or GOF variant, will result in high plasma levels of active metabolites leading to increased side effects and/or efficacy. Thus, patients carrying CYP2C19*17 GOF variants have increased conversion of clopidogrel to active metabolites resulting in reduced cardiovascular events and/or increased bleeding episodes [24-33]. Co-administration of an inducer of CYP1A2, CYP2C9, and/or CYP3A4 would be expected to result in greater efficacy of clopidogrel, with increased risk of bleeding, however no studies have been published to establish this. 
Fig. 1 The predicted active drug/ active metabolites of prodrugs plasma levels and biliary excretion changes without or with the presence of inhibitors or LOF variants or both on metabolizing enzymes. The predicted active drug/active metabolites of prodrugs plasma levels and biliary excretion changes without (a-1/a-2) or with the presence of inhibitors or LOF variants $(\mathbf{b}-\mathbf{1} / \mathbf{b}-\mathbf{2})$ or both (c-1/c-2) on metabolizing enzymes. a-1/a-2 represent the normal scenario with no interacting drug or genetic variant. In b-1/b-2 either an inhibitory drug or loss-offunction variant (LOF) in the metabolizing enzyme, results in reduced metabolism to inactive metabolites, and increased (b-1)/ decreased (b-2) active drug in the systemic circulation. In c-1/ c-2 the presence of inhibitory drug and the LOF genetic variant combine to produce greater increase (c-1)/decrease (c-2) in the systemic concentration of active drug
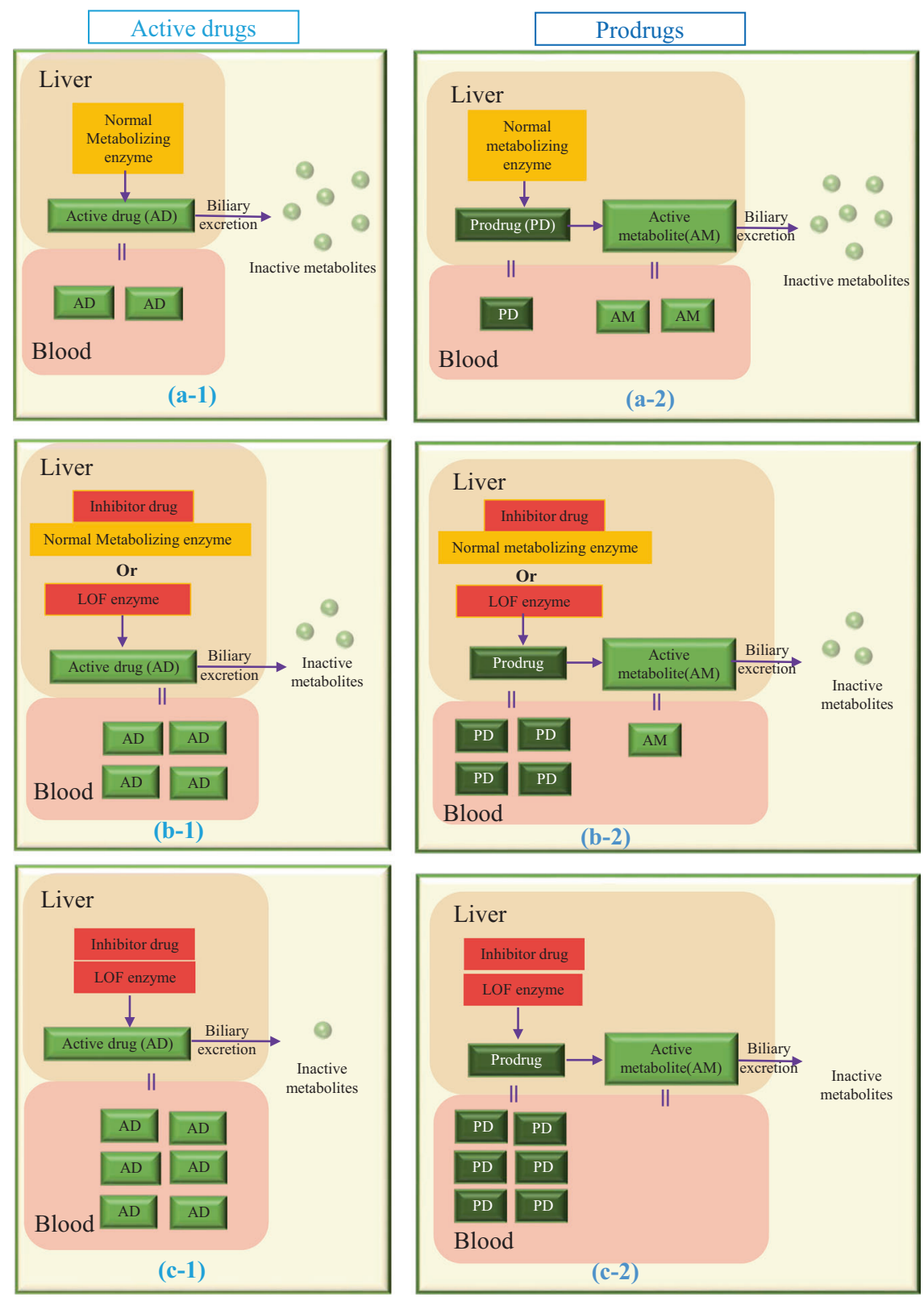

Figure 2 shows the predicted changes of plasma levels of active drugs and active metabolites of prodrugs with and without the presence of inducers and/or GOF variants.

\section{Phenoconversion interactions}

As described above, a temporary phenotype shift can be seen when the perpetrator drug and genetic effect are opposed. For example, the presence of reduced function CYP2C9 variants results in reduced tolbutamide (a CYP2C9 substrate) metabolism, yet co-treatment with rifampicin (a CYP2C9 inducer) in these patients reverses this genetic effect resulting in a twofold increase in tolbutamide clearance [34]. Conversely, proton pump inhibitors
(CYP2C19 inhibitors) treatment with clopidogrel results in phenoconversion in genetically determined ultra-rapid phenotype to a poor metabolizer status indicated by loss of clopidogrel efficacy [35].

The beneficial side of phenoconversion interactions is that genetically determined phenotypes can be normalized by the addition of medications of opposite effects on metabolism. For example, resistance to nortriptyline (CYP2D6 substrate) due to abnormally rapid metabolism has been successfully reversed and normalized with the addition of paroxetine a (CYP2D6 inhibitor), which produces a recovery of nortriptyline therapeutic plasma levels [36].

Figure 3 presents different scenarios of phenoconversion interactions. 
Fig. 2 The predicted active drug/ active metabolites of prodrugs plasma levels and biliary excretion changes with out or with the presence of inducers or GOF variants or both on metabolizing enzymes. The predicted active drug/active metabolites of prodrugs plasma levels and biliary excretion changes without (a-1/a-2) or with the presence of inducers or GOF variants (b-1/b-2) or both (c-1/c-2) on metabolizing enzymes. a-1/a-2 represent the normal scenario with no interacting drug or genetic variant. In $\mathbf{b}-\mathbf{1} / \mathbf{b}-\mathbf{2}$ either an inducer drug or gain-of-function variant $(\mathrm{GOF})$ in the metabolizing enzyme, results in increased metabolism to inactive metabolites, and decreased (b1)/increased (b-2) active drug in the systemic circulation. In c-1/ c-2 the presence of inducer drug and the GOF genetic variant combine to produce greater decrease(c-1)/increase(c-2) in the systemic concentration of active drug
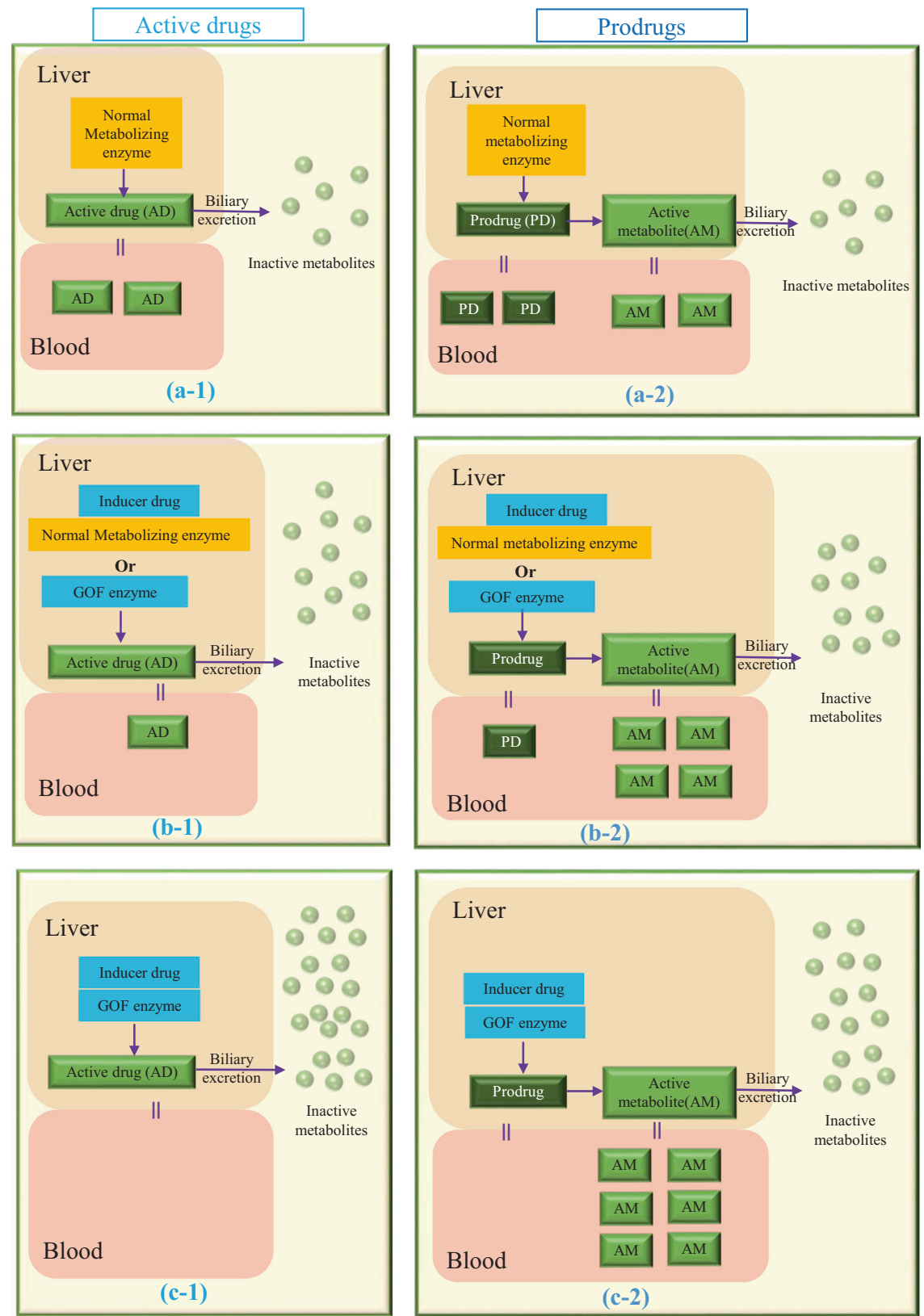

Drug-drug-transporters genes interactions (DDTGIs)

Drug transporters govern the movement of pharmaceutical compounds from and into different body tissues. The liver, kidney, blood-brain barrier (BBB), and intestine are the key sites of transporters that influence drug PK. In addition to summarizing the distribution and localization of transporters, Fig. 4 also classifies transporters into three categories according to the similarity of transport directions in different tissue types (the figure has been formulated with the aid of reference [37]). Drug-drug-gene interactions for transporters are less well studied than for metabolizing enzymes. For each subgroup, Drug Transporter-gene interaction
(DTGI) studies will be utilized (if no direct DDTGI studies are available) to illustrate each mechanism for potential interaction. Similar to the drug metabolizing enzyme scenarios outlined above, we predict that these interactions may be intensified or reversed, via inhibitory/induction or phenoconversion pathways, with the co-administration of inhibitors or inducers.

\section{Efflux transporters}

Efflux transporters have been classified into two groups (group I and group II) according to the similarity in the transport directions. 


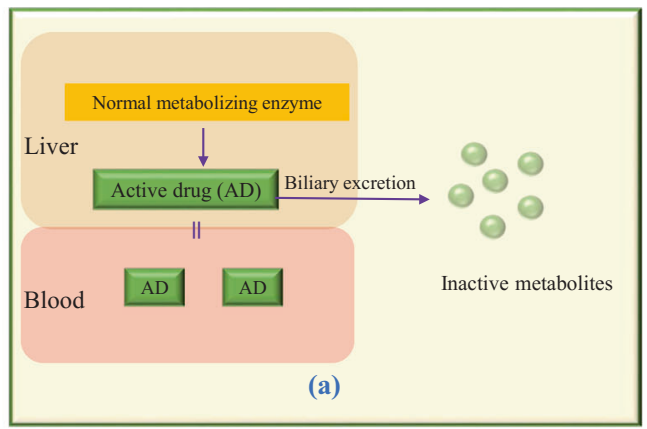

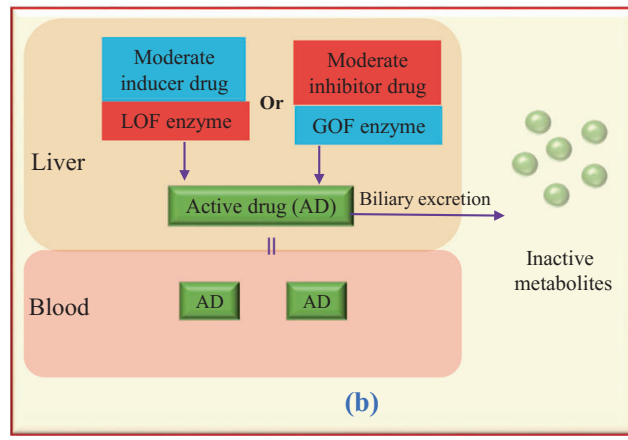

Fig. 3 Different scenarios of phenoconversion interactions where genetic effects may be reversed or shifted in the opposite direction. a Represents the normal scenario with no interacting drug or genetic variant. In b the effect of loss-of-function variant (LOF) or gain-offunction variant (GOF) is reversed with the presence of a moderate inducer drug or a moderate inhibitor drug respectively and results in a clinical outcome similar to the normal situation (a). In $\mathbf{c}$ the presence

Group I P-glycoprotein 1 (P-gp, ABCB1), multidrug resistance-associated protein 2 (MRP2, $\mathrm{ABCC} 2$ ), and breast cancer resistant protein (BCRP, ABCG2) transporters are expressed in the intestine, liver, kidney, and BBB, sharing similar transport pathways. They efflux substrates back to intestinal lumen, facilitate hepatic and renal excretion (excluding BCRP), and work inversely in the BBB where they protect the brain from the entry of xenobiotics and return them back to systemic circulation. Blocking their function in the intestine, liver, or kidney is expected to elevate a substrate's systemic exposure (although opposite effects would be predicted if inhibiting transport across the BBB).

In this group, the most evidence for DDTGI comes from drugs altering $\mathrm{ABCB} 1$ (P-gp) transport and genetic variants in the gene encoding this transporter. For example, cyclosporine is an ABCB1 substrate. Diltiazem (a moderate ABCB1 inhibitor [38]) has been shown to increase cyclosporin trough concentrations in Chinese patients who carry the TT genotype (low P-gp activity) at rs 1045642 $(\mathrm{C}>\mathrm{T})$ in $\mathrm{ABCB} 1$; yet no effect was seen in other ABCB1 genotypes (e.g., $C C$ at rs 1045642) [39]. Methadone is also a P-gp substrate, acting in the brain and effluxed across the BBB via P-gp. Patients with the TT genotype at rs1045642 and treated with quetiapine (ABCB1 inhibitor) experienced the lowest increase in methadone plasma levels compared with those with CT or CC genotypes (3\% vs $23 \%$ vs $33 \%$ respectively) [40]. Low methadone plasma levels in this study would be explained by loss of the ABCB1 protective function in the $\mathrm{BBB}$ which results in increased intracerebral concentration of this central nervous system (CNS) drug. As a result of a similar DDTGI mechanism, the CNS drug granisetron was associated with increased efficacy in Japanese subjects (Supplementary Table 1) [41].

In some cases, it seems that adding strong inhibitors abolishes the effect of genotype. For example, no additional inhibitory effects were detected in carriers of different genotypes of the rs1045642 (C>T) ABCB1 variant who were either on dabigatran/rivaroxaban-clarithromycin combination or tacrolimus-itraconazole combination (ABCB1 substrates-ABCB1 strong inhibitors [38]) [42, 43].

$\mathrm{ABCC} 2$ and $\mathrm{ABCG} 2$ would be predicted to follow similar interaction scenarios as $\mathrm{ABCB} 1$, yet we were unable to find any studies that report DDTGIs for these transporters.

Group II Unlike group I transporters, there are no published studies describing DDTGIs for group II transporters. So here we report DGTIs to highlight the potential mechanisms whereby genes and drugs that alter these transporters may influence drug outcomes. MRP1(ABCC1), MRP3 (ABCC3), and MRP4(ABCC4) share the similar transport direction in the kidney and $\mathrm{BBB}$ as the Group I 

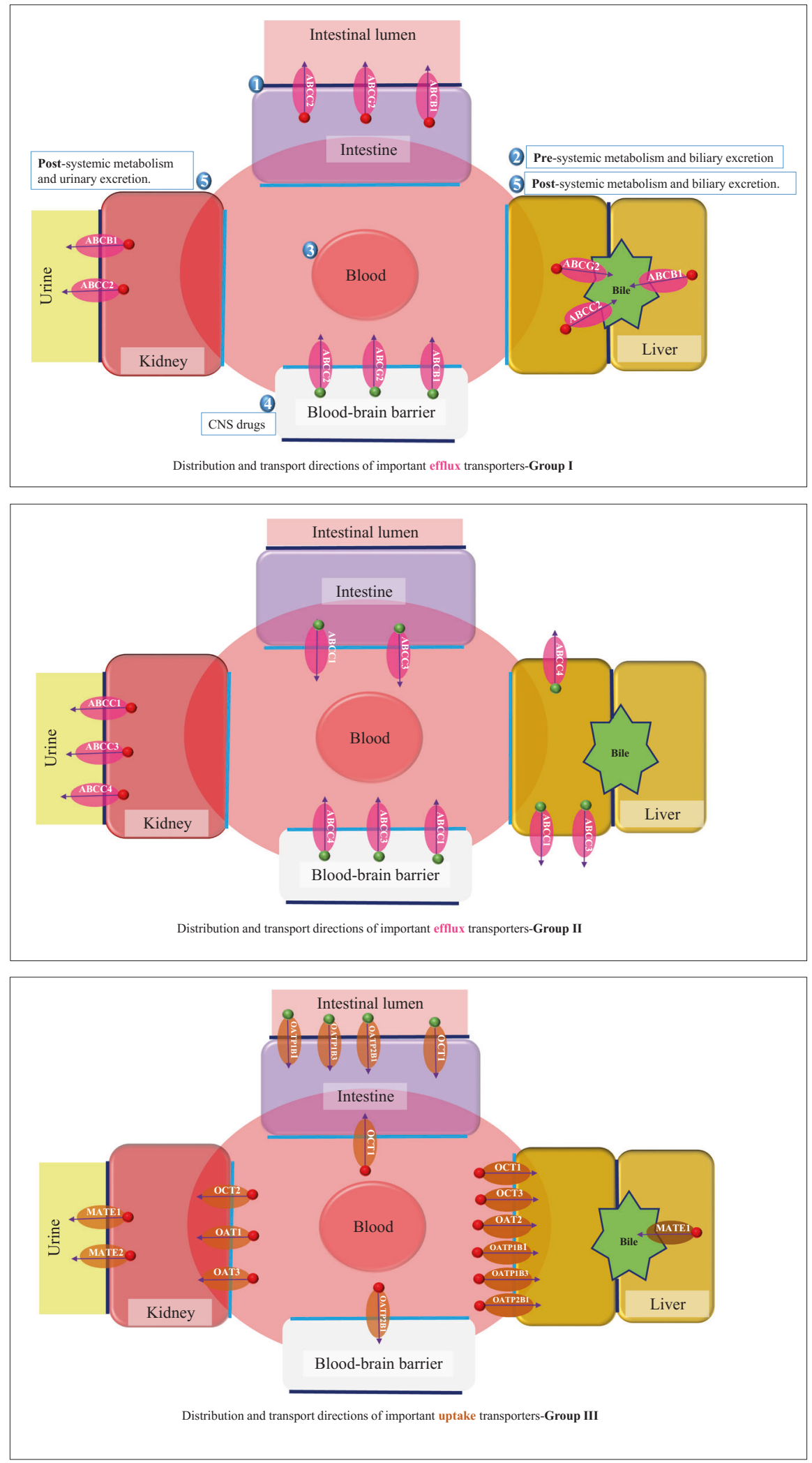

Fig. 4 Drug transporters as classified into three categories according to the similarity of the transport directions in different tissue types. Numbers from $(1)$ to $\mathbf{5}=$ order of oral drug movement through different tissue types. Nonoral drug formulations bypass the effect of intestinal transporters. $\mathrm{O} / \mathrm{O}=$ increased/decreased substrate drug plasma level is predicted as a result of impairment of this transporter due to LOF variants or inhibitors. The reverse is predicted with GOF variants or inducers. The presence of the two factors (i.e. LOF variant + an inhibitor or GOF variant + an inducer) is predicted to double the clinical impact with neutralizing or shifting the clinical effect when the preparator drug and genetic effect are opposed (phenoconversion interactions). = Apical membrane. $=$ Basolateral membrane 
transporters. However, in the liver, they are expressed in the basolateral membrane working to pump drugs back into systemic circulation. MRP1, for example, transports the active metabolite of irinotecan ( $\mathrm{SN}-38)$ out of hepatocytes into the blood contributing to the well-known side effect of irinotecan induced neutropenia [44]. The reduced function variant, rs17501331, in the $A B C C 1$ gene is associated with low incidence of neutropenia; the reverse effect was detected with the GOF variant rs6498588 in the same gene [45]. In some cases, increased activity of the MRP1 transporter can be advantageous, as seen with methotrexate hepatotoxicity where carriers of wild-type genotype of $A B C C 1$ rs246240 $(\mathrm{A}>\mathrm{G})$ variant are at higher risk for developing methotrexate toxicity compared with carriers of reduced function alleles [46]. Of note, MRP1 is also expressed in the myocardium protecting the heart from the entry of xenobiotics [47]. For example, the reduced transport associated with the rs45511401 $(\mathrm{G}>\mathrm{T})$ in $\mathrm{ABCC} 1$ increases the chance of developing cardiotoxicity due to intracellular accumulation of doxorubicin [48]. MRP1 and MRP3, in contrast to P-gp, MRP2 and BCRP, are expressed in the basolateral membrane of the intestine effluxing substrates into the portal circulation. As orally administered drugs are first exposed to intestinal transporters, any modification of their role might affect drug concentration in the other tissues (liver, kidney, or BBB). C. $1037 \mathrm{C}>\mathrm{T}$ and c. $1820 \mathrm{G}>\mathrm{A}$ ABCC3 variants, for example, have low transport activity [49] suggesting their potential to diminish the bioavailability of oral MRP3 substrates irrespective of subsequent alteration in transport into other tissues, or subsequent metabolism.

\section{Uptake Transporters (Group III)}

In the liver, kidney, and BBB, all important uptake transporters (organic cation transporters (OCTs) $1 / 2 / 3$, organic anion-transporting polypeptide (OATP) 1B1/1B3/2B1, and multidrug and toxic compound extrusion proteins (MATE) 1/2), follow an identical main route for transporting their substrates: from systemic circulation into different tissues or urine/bile in case of MATEs. Consequently, reducing or increasing these transport capacities would result in increased or reduced systemic drug concentrations respectively. The reverse effects are seen with the uptake transporters expressed in the intestinal apical membrane such as OATPs and OCT1 since the transportation pathway is in the opposite direction.

In some circumstances, altering uptake transporter function can increase ADRs. For example, it has been observed that carriers of two OCT1 (SLC22Al) reduced function alleles who were treated with OCT1 inhibitors were over four times more likely to develop gastrointestinal side effects with metformin (an OCT1 substrate) treatment, which would be attributable to metformin accumulation in the intestinal lumen (assuming apical OCT1 localization) [50]. This finding was supported by a previous study [51]. At the level of renal uptake transporters, other DDTGIs have been reported in which carrying the mutant alleles and the co-administration of inhibitors was linked to increased metformin plasma levels/toxicity or reduced clearance (see Supplementary Table 1) [52, 53]. By contrast, reducing transport in some cases may reduce certain side effects. For instance, cisplatin (a OCT2 (SLC22A2) substrate) is both a nephrotoxic and an ototoxic agent. People carrying the rs316019 (C>A) OCT2 mutation were protected from these adverse reactions as the variant resulted in reduced transport of cisplatin into the kidney and the inner ear (cochlea) (where OCT2 is expressed as well) [54-56].

In many situations, the efficacy of a drug relies upon the ability of that drug to access certain tissues. Statins are taken up into the liver by OATP1B1(SLCOIBI) and this is crucial for their lipid lowering effect. Reducing this uptake pathway reduces statin efficacy and raises plasma concentrations, resulting in myopathy and, rarely, rhabdomyolysis. The rs4149056 (T>C) (SLCO1B1*15) variant has been widely studied, and in 23 studies [57-79], this variant has been persistently connected to increased statin plasma exposure, muscle aches, dose reduction, and/or treatment-resistant phenotypes. A number of other DDGIs have been described for the SLCO1B1 transporter. For example, although the increase in pravastatin (SLCO1B1 substrate) AUC after treatment with ritonavir (SLCO1B1 inhibitor) was not statistically significant (21\% increase vs pravastatin alone) a large interaction was seen in those carrying the SLCO1B $1 * 15$ or $* 17$ haplotypes, with a resulting $113 \%$ elevation in pravastatin AUC [80]. Other DDTGIs with the similar mechanism have also been published (see Supplementary Table 1) [81-83]. Interestingly, unlike the ritonavir example just outlined, in some situations reduced function variants do not show any significant PK change until after the addition of inhibitors. For example, patients with AG or AA genotypes at rs2289669(G>A) of the MATE1 transporter only had significantly lower metformin (MATE1 substrate) clearance compared with carriers of GG genotype after treatment with ranitidine (a MATE1 inhibitor) [84].

\section{DDGls and challenges in clinical practice}

Metabolizing enzyme and transporter substrates, inducers, or inhibitors are not fully documented in many popular drug interaction databases, leaving physicians unaware of potentially important interactions. In addition, most of the resources commonly used by prescribers (e.g., Stockley's, Micromedex, Drug.com, RxList, or other drug interaction 
checkers) do not consider genetic variation when classifying drug interactions into minor, moderate, or major classes. Genetic variation may markedly increase or ameliorate the severity of potential drug interactions and do need to be considered when considering real-world use of drugs.

This review has discussed the different mechanisms of interactions in their simplest forms with the assumption that the patient is free of transporter polymorphisms or inhibitors/ inducers in the case of discussing DDMEGIs and vice versa with DDTGIs. However, in real-world clinical practice, achieving precisely tailored drug therapy requires a detailed examination of all mutations in the candidate enzyme or transporter genes with good awareness of the entire prescribed medications and possible pathways of interaction. Thus, the clinical scenario ranges from a relatively simple picture where the effect of genotype and interacting drug(s) can be approximated and treatment altered accordingly, to a far more complex scenario where physiologically based PK (PBPK) modeling may be helpful and where evaluation of large scale clinical data linked to genotypes is required to evaluate the clinical impact of multiple interacting drugs/multiple genotypes on drug outcomes.

Consider a relatively simple scenario: a patient with type 2 diabetes treated with metformin (has no effect on CYPs) who carries reduced function variants in CYP2C9 $(* 2$ or *3 variants) and who is started on gliclazide (CYP2C9/19 substrate). Reduced metabolism of gliclazide will result in increased efficacy [85] and increased risk of hypoglycemia [86]. The metformin use will not alter this DGI. However, if this patient were also treated with pioglitazone and/or atorvastatin (both are CYP2C9/19 inhibitors) they would be at potentially even greater risk of gliclazide-induced hypoglycemia and should be treated with a reduced dose of gliclazide. However, even for this simple scenario, such DDGI studies have not been reported; nor have dosing algorithms been developed to date for patients with CYP2C9 variants prescribed sulphonylureas and as such it is difficult to implement this into drug interaction calculators.

There are many more complex scenarios where, for example, a combination of both metabolizing enzyme and transporter LOF/GOF variants, as well as inhibitors/inducers are included. This kind of interaction may be only initially predictable when all their subinteractions result in the same clinical effect. For instance, reduced CYP3A4 and SLCO1B1 activities can both result in increased AUC of the substrate drug and a greater harm would be anticipated. Carriers of the TC genotype of SLCO1B1 rs4149056 (T>C) variant who are treated with amlodipine (CYP3A4 inhibitor) experienced a 90\% increased simvastatin AUC compared with subjects not treated with amlodipine and wild-type for rs4149056 [87]. A similar scenario was reported with other two case reports (see Supplementary Table 1) [88, 89].
In other situations, subinteractions do not share a similar clinical effect. Here, predicting the overall clinical outcome is challenging. As an illustration, oral rosuvastatin is mainly eliminated via biliary excretion with a minor contribution of CYP2C9 to its metabolism [90]. This implies that its transporters (e.g., $\mathrm{ABCC} 2, \mathrm{ABCG} 2, \mathrm{ABCC} 1$, and SLCO1B1) are the core players in its elimination. The concomitant administration of verapamil (an ABCC1/2 inhibitor) and venlafaxine (an ABCG2 inducer) in those who have inherited CYP2C9*3 and/or SLCO1B1 rs4149056 ( $\mathrm{T}>\mathrm{C})$ LOF variants results in unpredictable clinical consequences. CYP2C9, SLCO1B1, and ABCC2 impairment would boost rosuvastatin AUC, inducing ABCG2 would lower rosuvastatin AUC, and inhibition of ABCC 1 could result in both increase or decrease in AUC (high AUC if the site of interaction is in the kidney and low AUC if it is in the intestine or liver). The exact estimation of the predicted net AUC following a certain DDGI relies on calculating the contribution of each metabolizing enzyme and transporter to the elimination process (i.e. degree of sensitivity of substrates), inhibition/induction potency of the perpetrator agent or the net effect of multiple inhibitors, inducers, or both, and the net percentage of reduction/elevation in the enzyme/s and/or transporter/s activity caused by a single or more SNPs. The outcome of such a hugely complex scenario is impossible to predict by the clinician, and requires a clinical support tool based upon a PK DDGI prediction algorithm. Most of the current work concentrates on generating either DD or DG interaction predictors rather than the combined effect of both drugs and variants. However, using PBPK models, one predictor tool (https://www.ddi-predictor.org/) has recently been successfully generated to estimate drug exposure and the recommended dose following the dual action of both the perpetrator drug and mutations in certain CYPs (CYP2D6, CYP2C9, and CYP2C19) [91]. Other PBPK models do attempt to incorporate genotype and drug-drug interactions, but these do not model transporter variants well and have yet to translate through into clinically useful tools [92].

An alternative method to evaluate the impact of DDGIs is via metabolizing enzymes and transporters endogenous biomarkers rather than plasma concentrations of substrate drugs. Multiple enzymes/transporters-related biomarkers have been identified [93]. For instance, it has been shown that the cholesterol, cortisone, and cortisol metabolites: $4 \beta$-hydroxycholestrol, $6 \beta$-hydroxycortisone, and $6 \beta$ hydroxycortisole, respectively, which are catalyzed by CYP3A4 activity, are increased under the effect of inducers and decreased with inhibitors of CYP3A4. It was also recognized that bufotenine is a major metabolite resulting from the metabolizing activity of CYP2D6. With regard of transporters, several studies have observed the association between increased bilirubin plasma levels and 
reduced hepatic OATP1B1/1B3 uptake function. The similar scenario was noted recently with the novel biomarkers coproporphyrins I and III (CPs I and III) where plasma CPs levels elevated with the inhibition of these transporters to a similar extent as with rosuvastatin. In DDGIs studies, endogenous biomarkers can be utilized to predict the effect of both genetic variants and inhibitors/ inducers on the substrate drugs plasma levels.

It is worth noting that potential DDIs do not necessarily reflect actual interactions. It has been observed that clinically significant interactions are consistently lower than theoretically predictable interactions [94]. However, the authors noted that $20 \%$ of ADRs are linked with DDIs; most of them are serious with a high percentage of fatal cases. They also concluded that therapeutic failure secondary to DDIs, which is usually underestimated, represents a considerable part of total DDIs-related undesirable effects. The degree of clinical significance can be judged by observing other risk factors associated with a potential DDI such as polypharmacy and genetic variants. Polypharmacy is commonly seen with elderly and hospitalized patients making them the most vulnerable patient's subgroups to clinically significant interactions besides carriers of risky genetic variants. In addition, not all types of DDGIs are expected to be common. Induction and phenoconversion DDGIs are predicted to be seen with lower incidence compared with inhibitory DDGIs as the majority of perpetrator drugs are inhibitors rather than inducers and most of functional genetic variants are loss rather than GOF mutations.

The increasing availability of 'big data' linking health data and genomics has the potential to evaluate the realworld clinical impact of multiple drugs/multiple variant interactions. A number of data sets are now available or about to become available for study. In Scotland national prescribing and linked outcomes are available for the entire population enabling evaluation of real-world DDIs, and with an increasing bioresource (https://www.registerforsha re.org) it should be possible to evaluate DDGIs in $\sim 500 \mathrm{~K}$ people over the next few years. In addition, other resources such as UK biobank including genetic information on $500 \mathrm{~K}$ individuals (with primary care data available on $200 \mathrm{k}$ during 2018) and other national bioresources (such as the Danish biorepository) and US bioresources linked to EHRs (EMERGE network) will enable the evaluation of n-way DDG interactions to identify clinically important interactions that can be incorporated into clinical decision support tools in the future.

\section{Conclusion}

Dozens of new pharmaceutical compounds enter the market each year and a considerable number of patients are prescribed multiple drugs that necessitate the utilization of drug interaction databases for better management. One of the major limitations of these drug interaction checkers is the omission of the genetic effect on drug interactions. This reflects both the lack of clinical studies that quantity potential DDGIs and the fact that genetic information is rarely available on patients at the point of prescribing. This review has illustrated, with some examples, various mechanisms by which DDGIs can occur at the level of metabolizing enzymes, drug transporters, or both (this has been summarized in Supplementary Table 1). We have also shown the different degrees of complexity clinicians may face in judging the predicted clinical outcome following a certain DDGI. The more factors that are included, the more challenging it becomes to evaluate the outcome. There is a need for PBPK models, clinical studies and real-world evaluation of drug outcomes linked to genetic information to develop clinical useful DDGI models, to reduce adverse DDIs and improve drug outcomes in the setting of increasing multi-morbidity and polypharmacy.

\section{Compliance with ethical standards}

Conflict of interest There is no conflict of interest to disclose that could be perceived to bias our work. This work is a part of an entire $\mathrm{PhD}$ project which is supported by the main author's sponsor for the purpose of completing his $\mathrm{PhD}$ degree. Authors and the sponsor have no conflict of interest to disclose.

Publisher's note Springer Nature remains neutral with regard to jurisdictional claims in published maps and institutional affiliations.

Open Access This article is licensed under a Creative Commons Attribution 4.0 International License, which permits use, sharing, adaptation, distribution and reproduction in any medium or format, as long as you give appropriate credit to the original author(s) and the source, provide a link to the Creative Commons license, and indicate if changes were made. The images or other third party material in this article are included in the article's Creative Commons license, unless indicated otherwise in a credit line to the material. If material is not included in the article's Creative Commons license and your intended use is not permitted by statutory regulation or exceeds the permitted use, you will need to obtain permission directly from the copyright holder. To view a copy of this license, visit http://creativecommons. org/licenses/by/4.0/.

\section{References}

1. Lazarou J, Pomernaz B, Corey P. Incidence of adverse drug reactions in hospitalized patients: a meta-analysis of prospective studies. Surv Anesthesiol. 1999;43:53-4.

2. Bouvy J, De Bruin M, Koopmanschap M. Epidemiology of adverse drug reactions in europe: a review of recent observational studies. Drug Saf 2015;38:437-53.

3. MiDatabank Adverse Drug Reaction (ADR) reporting - 2016 update, SPS-Specialist Pharmacy Service - The first stop for professional medicines advice. 2017. Accessed 6 Sep 2017. https://www.sps.nhs.uk/articles/midatabank-adverse-drug-rea ction-adr-reporting-2016-update/. 
4. Exploring the costs of unsafe care in the NHS. frontier-economics. 2014. Accessed 11 Sep 2017. http://www.frontier-economics. com/documents/2014/10/exploring-the-costs-of-unsafe-care-inthe-nhs-frontier-report-2-2-2-2.pdf.

5. Howard R, Avery A, Slavenburg S, Royal S, Pipe G, Lucassen P, et al. Which drugs cause preventable admissions to hospital? A systematic review. Br J Clin Pharmacol. 2007;63:136-47.

6. Rodrigues M, Oliveira C. Drug-drug interactions and adverse drug reactions in polypharmacy among older adults: an integrative review. Rev Lat Am de Enferm. 2016;24:e2800.

7. Verbeurgt P, Mamiya T, Oesterheld J. How common are drug and gene interactions? Prevalence in a sample of 1143 patients with CYP2C9, CYP2C19 and CYP2D6 genotyping. Pharmacogenomics 2014;15:655-65.

8. Bahar M, Setiawan D, Hak E, Wilffert B. Pharmacogenetics of drug-drug interaction and drug-drug-gene interaction: a systematic review on CYP2C9, CYP2C19 and CYP2D6. Pharmacogenomics 2017;18:701-39.

9. Andersson M, Eliasson E, Lindh J. A clinically significant interaction between warfarin and simvastatin is unique to carriers of theCYP2C9*3allele. Pharmacogenomics 2012;13:757-62.

10. Malhi H. Warfarin and celecoxib interaction in the setting of cytochrome P450 (CYP2C9) polymorphism with bleeding complication. Postgrad Med J. 2004;80:107-9.

11. Uno T, Shimizu M, Yasui-Furukori N, Sugawara K, Tateishi T. Different effects of fluvoxamine on rabeprazole pharmacokinetics in relation to CYP2C19 genotype status. Br J Clin Pharmacol. 2006;61:309-14.

12. Yasui-Furukori N, Saito M, Uno T, Takahata T, Sugawara K, Tateishi T. Effects of fluvoxamine on lansoprazole pharmacokinetics in relation to CYP2C19 genotypes. J Clin Pharmacol. 2004;44:1223-9.

13. Cho J, Yu K, Jang I, Yang B, Shin S, Yim D. Omeprazole hydroxylation is inhibited by a single dose of moclobemide in homozygotic EM genotype for CYP2C19. Br J Clin Pharmacol. 2002;53:393-7.

14. Hamelin B. Significant interaction between the nonprescription antihistamine diphenhydramine and the CYP2D6 substrate metoprolol in healthy men with high or low CYP2D6 activity. Clin Pharmacol Therapeutics. 2000;67:466-77.

15. Damy T, Pousset F, Caplain H, Hulot J, Lechat P. Pharmacokinetic and pharmacodynamic interactions between metoprolol and dronedarone in extensive and poor CYP2D6 metabolizers healthy subjects. Fundam Clin Pharmacol. 2004;18:113-23.

16. Zhu L, Brüggemann R, Uy J, Colbers A, Hruska M, Chung E, et al. CYP2C19 Genotype-Dependent Pharmacokinetic Drug Interaction Between Voriconazole and Ritonavir-Boosted Atazanavir in Healthy Subjects. J Clin Pharm. 2017;57:235-46.

17. Imamura $\mathrm{C}$, Furihata $\mathrm{K}$, Okamoto $\mathrm{S}$, Tanigawara $\mathrm{Y}$. Impact of cytochrome $\mathrm{P} 450$ 2C19 polymorphisms on the pharmacokinetics of tacrolimus when coadministered with voriconazole. J Clin Pharmacol. 2015;56:408-13.

18. Shi H, Yan J, Zhu W, Yang G, Tan Z, Wu W, et al. Effects of erythromycin on voriconazole pharmacokinetics and association with CYP2C19 polymorphism. Eur J Clin Pharmacol. 2010;66:1131-6.

19. Saito M, Yasui-Furukori N, Uno T, Takahata T, Sugawara K, Munakata A, et al. Effects of clarithromycin on lansoprazole pharmacokinetics between CYP2C19 genotypes. Br J Clin Pharmacol. 2005;59:302-9.

20. Sangkuhl K, Klein T, Altman R. Clopidogrel pathway. Pharmacogenetics Genomics. 2010;20:463-5.

21. Furuta T, Iwaki T, Umemura K. Influences of different proton pump inhibitors on the anti-platelet function of clopidogrel in relation to CYP2C19 genotypes. Br J Clin Pharmacol. 2010;70:383-92.
22. Harmze A, Van Werkum J, Souverein P, Breet N, Bouman H, Hackeng $\mathrm{C}$, et al. Combined influence of proton-pump inhibitors, calcium-channel blockers and CYP2C19*2 on on-treatment platelet reactivity and on the occurrence of atherothrombotic events after percutaneous coronary intervention. J Thrombosis Haemost. 2011;9:1892-901.

23. Malingré M, Godschalk P, Klein S. A case report of voriconazole therapy failure in a homozygous ultrarapid CYP2C19*17/*17 patient comedicated with carbamazepine. Br J Clin Pharmacol. 2012;74:205-6.

24. Carlquist J, Knight S, Horne B, Huntinghouse J, Rollo J, Muhlestein J, et al. Cardiovascular risk among patients on clopidogrel anti-platelet therapy after placement of drug-eluting stents is modified by genetic variants in both the CYP2C19 and ABCB1 genes. Thrombosis Haemost. 2013;109:744-54.

25. Chan M, Tan K, Tan H, Huan P, Li B, Phua Q, et al. CYP2C19 and $\mathrm{PON} 1$ polymorphisms regulating clopidogrel bioactivation in Chinese, Malay and Indian subjects. Pharmacogenomics 2012;13:533-42.

26. Harmsze A, van Werkum J, Hackeng C, Ruven H, Kelder J, Bouman $\mathrm{H}$, et al. The influence of CYP2C19*2 and $* 17$ on ontreatment platelet reactivity and bleeding events in patients undergoing elective coronary stenting. Pharmacogenetics Genomics. 2012;22:169-75.

27. Cayla G, Hulot J, O'Connor S, Pathak A, Scott S, Gruel Y, et al. Clinical, angiographic, and genetic factors associated with early coronary stent thrombosis. JAMA 2011;306:1765-74.

28. Gurbel P, Shuldiner A, Bliden K, Ryan K, Pakyz R, Tantry U. The relation between CYP2C19 genotype and phenotype in stented patients on maintenance dual antiplatelet therapy. Am Heart J. 2011;161:598-604.

29. Wallentin L, James S, Storey R, Armstrong M, Barratt B, Horrow $\mathrm{J}$, et al. Effect of CYP2C19 and ABCB1 single nucleotide polymorphisms on outcomes of treatment with ticagrelor versus clopidogrel for acute coronary syndromes: a genetic substudy of the PLATO trial. Lancet 2010;376:1320-8.

30. Sibbing D, Gebhard D, Koch W, Braun S, Stegherr J, Morath T, et al. Isolated and interactive impact of common CYP2C19 genetic variants on the antiplatelet effect of chronic clopidogrel therapy. J Thrombosis Haemost. 2010;8:1685-93.

31. Gladding P, Webster M, Zeng I, Farrell H, Stewart J, Ruygrok P, et al. The pharmacogenomics and pharmacodynamics of clopidogrel response: an analysis from the PRINC (Plavix Response in Coronary Intervention) trial. Heart, Lung Circulation. 2008;17: S82.

32. Dai Z. Relationship between cytochrome P450 2C19*17 genotype distribution platelet aggregation and bleeding risk in patients with blood stasis syndrome of coronary artery disease treated with clopidogrel. J Chin Integr Med. 2012;10:647-54.

33. Tiroch K, Sibbing D, Koch W, Roosen-Runge T, Mehilli J, Schömig A, et al. Protective effect of the CYP2C19*17 polymorphism with increased activation of clopidogrel on cardiovascular events. Am Heart J. 2010;160:506-12.

34. Vormfelde S, Brockmöller J, Bauer S, Herchenhein P, Kuon J, Meineke I, et al. Relative Impact of Genotype and Enzyme Induction on the Metabolic Capacity of CYP2C9 in Healthy Volunteers. Clin Pharmacol Therapeutics. 2009;86:54-61.

35. Depta J, Lenzini P, Lanfear D, Wang T, Spertus J, Bach R, et al. Clinical outcomes associated with proton pump inhibitor use among clopidogrel-treated patients within CYP2C19 genotype groups following acute myocardial infarction. Pharmacogenomics J. 2014;15:20-5.

36. Laine K, Tybring G, Hartter S, Andersson K, Svensson J, Widen $\mathrm{J}$, et al. Inhibition of cytochrome P4502D6 activity with paroxetine normalizes the ultrarapid metabolizer phenotype as measured 
by nortriptyline pharmacokinetics and the debrisoquin test. Clin Pharmacol Therapeutics. 2001;70:327-35.

37. Nicholls G, Youdim K. Drug transporters: volume 1: role and importance in adme and drug development, p.16. 54th edn. The Royal Society of Chmeistry (RSC) 2016; 2016. Accessed 9 Sep 2017. http://pubs.rsc.org/en/content/ebook/978-1-78262069-3.

38. Wessler J, Grip L, Mendell J, Giugliano R. The P-glycoprotein transport system and cardiovascular drugs. J Am Coll Cardiol. 2013;61:2495-502.

39. Wang Y, Li J, Wang X, Zhang Y, Wang C, Huang M. Diltiazem augments the influence of MDR1 genotype status on cyclosporine concentration in Chinese patients with renal transplantation. Acta Pharmacologica Sin. 2015;36:855-62.

40. Uehlinger C, Crettol S, Chassot P, Brocard M, Koeb L, BrawandAmey $\mathrm{M}$, et al. Increased (R)-methadone plasma concentrations by quetiapine in cytochrome $\mathrm{P} 450 \mathrm{~s}$ and $\mathrm{ABCB} 1$ genotyped patients. J Clin Psychopharmacol. 2007;27:273-8.

41. Tsuji D, Kim Y, Nakamichi H, Daimon T, Suwa K, Iwabe Y, et al. Association of ABCB1 polymorphisms with the antiemetic efficacy of granisetron plus dexamethasone in breast cancer patients. Drug Metab Pharmacokinetics. 2013;28:299-304.

42. Gouin-Thibault I, Delavenne X, Blanchard A, Siguret V, Salem J, Narjoz $\mathrm{C}$, et al. Interindividual variability in dabigatran and rivaroxaban exposure: contribution of $\mathrm{ABCB} 1$ genetic polymorphisms and interaction with clarithromycin. $\mathrm{J}$ Thrombosis Haemost. 2017;15:273-83.

43. Togashi M, Niioka T, Komatsuda A, Nara M, Okuyama S, Omokawa A, et al. Effect of CYP3A5 and ABCB1 polymorphisms on the interaction between tacrolimus and itraconazole in patients with connective tissue disease. Eur J Clin Pharmacol. 2015;71:1091-7.

44. Lowenberg D, Thorn C, Whirl-Carrillo M, Ramirez J, Gong L, Marsh S et al. Irinotecan Pathway, Pharmacokinetics. PharmGKB. Accessed 9 Oct 2017. https://www.pharmgkb.org/pathway/ PA2001.

45. Li M, Seiser E, Baldwin R, Ramirez J, Ratain M, Innocenti F, et al. ABC transporter polymorphisms are associated with irinotecan pharmacokinetics and neutropenia. Pharmacogenomics J. 2016. https://doi.org/10.1038/tpj.2016.75.

46. Warren R, Smith R, Campalani E, Eyre S, Smith C, Barker J, et al. Genetic variation in efflux transporters influences outcome to methotrexate therapy in patients with psoriasis. J Investig Dermatol. 2008;128:1925-9.

47. Jungsuwadee $\mathrm{P}$, Nithipongvanitch R, Chen Y, Oberley T, Butterfield D,St, Clair D, et al. Mrp1 Localization and Function in Cardiac Mitochondria after Doxorubicin. Mol Pharmacol 2009;75:1117-26.

48. Wojnowski L. NAD(P)H oxidase and multidrug resistance protein genetic polymorphisms are associated with doxorubicin-induced cardiotoxicity. Circulation 2005;112:3754-62.

49. Kobayashi K, Ito K, Takada T, Sugiyama Y, Suzuki H. Functional analysis of nonsynonymous single nucleotide polymorphism type ATP-binding cassette transmembrane transporter subfamily $\mathrm{C}$ member 3 . Pharmacogenetics Genomics. 2008;18:823-33.

50. Dujic T, Zhou K, Donnelly L, Tavendale R, Palmer C, Pearson E. Association of organic cation transporter 1 with intolerance to metformin in type 2 diabetes: a GoDARTS study. Diabetes 2014;64:1786-93.

51. Ahlin G, Chen L, Lazorova L, Chen Y, Ianculescu A, Davis R, et al. Genotype-dependent effects of inhibitors of the organic cation transporter, OCT1: predictions of metformin interactions. Pharmacogenomics J. 2010;11:400-11.

52. Grün B, Kiessling M, Burhenne J, Riedel K, Weiss J, Rauch G, et al. Trimethoprim-metformin interaction and its genetic modulation by OCT2 and MATE1 transporters. Br J Clin Pharmacol. 2013;76:787-96.

53. Wang Z, Yin O, Tomlinson B, Chow M. OCT2 polymorphisms and in-vivo renal functional consequence: studies with metformin and cimetidine. Pharmacogenetics Genomics. 2008;18:637-45.

54. Filipski K, Mathijssen R, Mikkelsen T, Schinkel A, Sparreboom A. Contribution of organic cation transporter 2 (OCT2) to cisplatin-induced nephrotoxicity. Clin Pharmacol Therapeutics. 2009;86:396-402.

55. Spracklen T, Vorster A, Ramma L, Dalvie S, Ramesar R. Promoter region variation in NFE2L2 influences susceptibility to ototoxicity in patients exposed to high cumulative doses of cisplatin. Pharmacogenomics J. 2016. https://doi.org/10.1038/tpj. 2016.52.

56. Lanvers-Kaminsky C, Sprowl J, Malath I, Deuster D, Eveslage M, Schlatter E, et al. Human OCT2 variant c.808G $>$ T confers protection effect against cisplatin-induced ototoxicity. Pharmacogenomics 2015;16:323-32.

57. Choi H, Bae K, Cho S, Ghim J, Choe S, Jung J, et al. Impact of CYP2D6, CYP3A5, CYP2C19, CYP2A6, SLCO1B1, ABCB1, and $\mathrm{ABCG} 2$ gene polymorphisms on the pharmacokinetics of simvastatin and simvastatin acid. Pharmacogenet Genomics 2013;25:595-608.

58. Luzum J, Theusch E, Taylor K, Wang A, Sadee W, Binkley P, et al. Individual and combined associations of genetic variants in CYP3A4, CYP3A5, and SLCO1B1 with simvastatin and simvastatin acid plasma concentrations. J Cardiovascular Pharmacol. 2015;66:80-5.

59. Mirošević Skvrce N, Macolić Šarinić V, Šimić I, Ganoci L, Muačević Katanec D, Božina N. ABCG2 gene polymorphisms as risk factors for atorvastatin adverse reactions: a case-control study. Pharmacogenomics 2015;16:803-15.

60. Tsamandouras N, Dickinson G, Guo Y, Hall S, Rostami-Hodjegan A, Galetin A, et al. Development and application of a mechanistic pharmacokinetic model for simvastatin and its active metabolite simvastatin acid using an integrated population PBPK approach. Pharm Res 2014;32:1864-83.

61. Birmingham B, Bujac S, Elsby R, Azumaya C, Wei C, Chen Y, et al. Impact of ABCG2 and SLCO1B1 polymorphisms on pharmacokinetics of rosuvastatin, atorvastatin and simvastatin acid in Caucasian and Asian subjects: a class effect? Eur J Clin Pharmacol. 2015;71:341-55.

62. Tsamandouras N, Dickinson G, Guo Y, Hall S, Rostami-Hodjegan A, Galetin A, et al. Identification of the effect of multiple polymorphisms on the pharmacokinetics of simvastatin and simvastatin acid using a population-modeling approach. Clin Pharmacol Ther. 2014;96:90-100.

63. Ferrari M, Guasti L, Maresca A, Mirabile M, Contini S, Grandi A, et al. Association between statin-induced creatine kinase elevation and genetic polymorphisms in SLCO1B1, ABCB1 and ABCG2. Eur J Clin Pharmacol. 2014;70:539-47.

64. De Keyser C, Peters B, Becker M, Visser L, Uitterlinden A, Klungel $\mathrm{O}$, et al. The SLCO1B1 c.521T>C polymorphism is associated with dose decrease or switching during statin therapy in the Rotterdam Study. Pharmacogenetics Genomics. 2014;24:43-51.

65. DeGorter M, Tirona R, Schwarz U, Choi Y, Dresser G, Suskin N, et al. Clinical and pharmacogenetic predictors of circulating atorvastatin and rosuvastatin concentrations in routine clinical care. Circulation. 2013;6:400-8.

66. Carr D, O’Meara H, Jorgensen A, Campbell J, Hobbs M, McCann $\mathrm{G}$, et al. SLCO1B1 genetic variant associated with statin-induced myopathy: a proof-of-concept study using the clinical practice research datalink. Clin Pharmacol Ther. 2013;94:695-701.

67. Ulvestad M, Skottheim I, Jakobsen G, Bremer S, Molden E, Åsberg A, et al. Impact of OATP1B1, MDR1, and CYP3A4 
expression in liver and intestine on interpatient pharmacokinetic variability of atorvastatin in obese subjects. Clin Pharmacol Therap. 2012;93:275-82.

68. Marciante K, Durda J, Heckbert S, Lumley T, Rice K, McKnight $\mathrm{B}$, et al. Cerivastatin, genetic variants, and the risk of rhabdomyolysis. Pharmacogenet Genomics 2011;21:280-8.

69. Donnelly L, Doney A, Tavendale R, Lang C, Pearson E, Colhoun $\mathrm{H}$, et al. Common nonsynonymous substitutions in SLCO1B1 predispose to statin intolerance in routinely treated individuals with type 2 diabetes: A Go-DARTS Study. Clin Pharmacol Therapeutics. 2010;89:210-6.

70. Voora D, Shah S, Spasojevic I, Ali S, Reed C, Salisbury B, et al. The SLCO1B1*5 genetic variant is associated with statin-induced side effects. J Am Coll Cardiol. 2009;54:1609-16.

71. Link E, Parish S, Armitage J, Bowman L, Heath S, Matsuda F, et al. SLCO1B1 variants and statin-induced myopathy-a genomewide study. N. Engl J Med. 2008;359:789-99.

72. Ho R, Choi L, Lee W, Mayo G, Schwarz U, Tirona R, et al. Effect of drug transporter genotypes on pravastatin disposition in European- and African-American participants. Pharmacogenetics Genomics. 2007;17:647-56.

73. Pasanen M, Fredrikson H, Neuvonen P, Niemi M. Different effects of SLCO1B1 polymorphism on the pharmacokinetics of atorvastatin and rosuvastatin. Clin Pharmacol Ther. 2007;82:726-33.

74. Niemi M, Pasanen M, Neuvonen P. SLCO1B1 polymorphism and sex affect the pharmacokinetics of pravastatin but not fluvastatin. Clin Pharmacol Ther. 2006;80:356-66.

75. Igel M, Arnold K, Niemi M, Hofmann U, Schw ABM, Lutjohann $\mathrm{D}$, et al. Impact of the SLCO1B1 polymorphism on the pharmacokinetics and lipid-lowering efficacy of multiple-dose pravastatin. Clin Pharmacol Therapeutics. 2006;79:419-26.

76. Pasanen M, Neuvonen M, Neuvonen P, Niemi M. SLCO1B1 polymorphism markedly affects the pharmacokinetics of simvastatin acid. Pharmacogenetics Genomics. 2006;16:873-9.

77. Thompson J, Man M, Johnson K, Wood L, Lira M, Lloyd D, et al. An association study of 43 SNPs in 16 candidate genes with atorvastatin response. Pharmacogenomics J. 2005;5:352-8.

78. Mwinyi J. Evidence for inverse effects of OATP-C (SLC21A6) *5 and $* 1 \mathrm{~b}$ haplotypes on pravastatin kinetics. Clin Pharmacol Therapeutics. 2004;75:415-21.

79. Nishizato Y. Polymorphisms of OATP-C (SLC21A6) and OAT3 (SLC22A8) genes: consequences for pravastatin pharmacokinetics. Clin Pharmacol Ther. 2003;73:554-65.

80. Aquilante C, Kiser J, Anderson P, Christians U, Kosmiski L, Daily E, et al. Influence of SLCO1B1 polymorphisms on the drugdrug interaction between darunavir/ritonavir and pravastatin. J Clin Pharmacol. 2012;52:1725-38.
81. Suwannakul S, Ieiri I, Kimura M, Kawabata K, Kusuhara H, Hirota $\mathrm{T}$, et al. Pharmacokinetic interaction between pravastatin and olmesartan in relation to SLCO1B1 polymorphism. J Hum Genet. 2008;53:899-904.

82. Kalliokoski A, Backman J, Kurkinen K, Neuvonen P, Niemi M. Effects of gemfibrozil and atorvastatin on the pharmacokinetics of repaglinide in relation to SLCO1B1 polymorphism. Clin Pharmacol Ther. 2008;84:488-96.

83. He Y, Zhang W, Chen Y, Guo D, Tu J, Xu L, et al. Rifampicin alters atorvastatin plasma concentration on the basis of SLCO1B1 521T >C polymorphism. Clin Chim Acta. 2009;405:49-52.

84. Cho S, Chung J. The MATE1 rs2289669 polymorphism affects the renal clearance of metformin following ranitidine treatment. Int J Clin Pharmacol Ther. 2016;54:253-62.

85. Zhou K, Donnelly L, Burch L, Tavendale R, Doney A, Leese G, et al. Loss-of-Function CYP2C9 variants improve therapeutic response to sulfonylureas in type 2 diabetes: A Go-DARTS Study. Clin Pharmacol Ther. 2009;87:52-6.

86. Ragia G, Petridis I, Tavridou A, Christakidis D, Manolopoulos V. Presence of CYP2C9*3 allele increases risk for hypoglycemia in Type 2 diabetic patients treated with sulfonylureas. Pharmacogenomics 2009;10:1781-7.

87. Jiang F, Choi J, Lee J, Ryu S, Park Z, Lee J, et al. The influences of SLCO1B1 and ABCB1 genotypes on the pharmacokinetics of simvastatin, in relation to CYP3A4 inhibition. Pharmacogenomics 2017;18:459-69.

88. Meyer zu Schwabedissen H, Siegmund W, Kroemer H, Rollnik J. Creatine kinase elevation caused by a combination of fluvastatin and telmisartan in a patient heterozygous for the CYP2C $9 * 3$ and $\mathrm{ABCC} 2-24 \mathrm{C}>\mathrm{T}$ variants: a case report. BMC Res Notes. 2014;7:688.

89. Marusic S, Lisicic A, Horvatic I, Bacic-Vrca V, Bozina N. Atorvastatin-related rhabdomyolysis and acute renal failure in a genetically predisposed patient with potential drug-drug interaction. Int J Clin Pharm. 2012;34:825-7.

90. Rosuvastatin - DrugBank. Drugbank.ca. Accessed 9 Oct 2017. https://www.drugbank.ca/drugs/DB01098.

91. Tod M, Nkoud-Mongo C, Gueyffier F. Impact of genetic polymorphism on drug-drug interactions mediated by cytochromes: a general approach. AAPS J. 2013;15:1242-52.

92. Zhuang $X, \mathrm{Lu}$ C. PBPK modeling and simulation in drug research and development. Acta Pharmaceutica Sin B 2016;6:430-40.

93. Mariappan T, Shen H, Marathe P. Endogenous biomarkers to assess drug-drug interactions by drug transporters and enzymes. Curr Drug Metab. 2017;18:757-68.

94. Magro L, Moretti U, Leone R. Epidemiology and characteristics of adverse drug reactions caused by drug-drug interactions. Expert Opin Drug Saf. 2011;11:83-94. 